\title{
Real-time interferometric characterization of a PVA based photopolymer.
}

\author{
Andrés Márquez ${ }^{1}$, Sergi Gallego ${ }^{1}$, David Méndez ${ }^{1}$, Manuel Ortuño $^{1}$, Elena Fernández $^{2}$, \\ Mariela L. Álvarez ${ }^{1}$, Cristian Neipp ${ }^{1}$, Augusto Beléndez ${ }^{1}$, Inmaculada Pascual ${ }^{2}$ \\ ${ }^{1}$ Depto. de Física, Ingeniería de Sistemas y Teoría de la Señal, Universidad de Alicante, Spain \\ ${ }^{2}$ Departamento de Óptica, Farmacología y Anatomía, Universidad de Alicante, Spain \\ Tel.: +34-96-5903400 (ext. 2543); Fax: +34-96-5909750; E-mail: Andres.Marquez@ua.es
}

\begin{abstract}
Recently, we have proposed the application of interferometric techniques, both in transmission and in reflection, to characterize in real-time the modulation performance of the photopolymers. In this work we use this approach to characterize the optical modulation properties of a polyvinyl alcohol/acrylamide (PVA/AA) photopolymer. A double beam interferometer is constructed, both in transmission and in reflection, in combination with the setup to expose the recording material. Some benefits are provided by this approach: direct calculation of the properties of the material is possible, index and surface modulation can be decoupled, and additional information can be obtained since the results are not influenced by diffusion processes. With this scheme we mainly characterize the properties at very low spatial frequencies, which can be useful to analyze the applicability of holographic recording materials in another range of applications, such as recording of diffractive optical elements (DOEs). Comparison with the conventional holographic characterization shows significant differences.
\end{abstract}

Keywords: Photopolymers, holographic data storage, diffractive optical elements, interferometry, phase modulation.

\section{INTRODUCTION}

Photopolymer based recording materials have been widely studied for holographic applications ${ }^{1-4}$. Their good properties as high diffraction efficiencies, low noise, self-developing, easy preparation, high thickness, low cost, etc., make these holographic recording materials optimum candidates for many holographic devices such as holographic memories ${ }^{5-7}$. For these applications, good response of spatial frequencies, between 700 lines $/ \mathrm{mm}$ and 1500 lines $/ \mathrm{mm}$, is required. Among the different photopolymer compositions, polyvinyl-alcohol/acrylamide (PVA/AA) materials is one of the promising materials in these applications and they have been deeply analyzed in holography ${ }^{8-10}$ and data storage ${ }^{11}$.

Holographic recording materials are typically characterized in terms of their diffraction efficiency by registering diffraction gratings with high spatial frequencies (larger than 1000 lines $/ \mathrm{mm}$ ) by optical interference of two plane waves. In the last years this technique has been intensively used for the polyvinyl alcohol (PVA) based photopolymers produced by our research group, obtaining very relevant information for the polymerization-diffusion processes taking place in the material $^{12}$, for the stability and storage conditions ${ }^{13}$, etc. This has enabled to optimize the composition of the photopolymers for holographic data storage, holographic optical elements or Bragg image processing ${ }^{3,14}$.

To obtain the values for the modulation properties of the material, mainly the index modulation, non-linear fitting of multiparametric models, such as the Rigorous Coupled Wave Theory (RCWT) ${ }^{8}$, to the experimentally measured diffracted and/or transmitted intensity are applied. The values obtained for the modulation properties at high spatial frequencies (more than 500 lines $/ \mathrm{mm}$ ) contain both the influence of photopolymerization reaction and diffusion of the

Practical Holography XXII: Materials and Applications, edited by Hans I. Bjelkhagen, Raymond K. Kostuk, Proc. of SPIE Vol. 6912, 691217, (2008) · 0277-786X/08/\$18 · doi: 10.1117/12.763548 
components in the material between the exposed and unexposed zones ${ }^{15}$. Depending on the strength assumed in the models for the two processes (photopolymerization and diffusion) the values for the material parameters may largely vary. Therefore, it would be a clear advantage to have some technique at hand so as to measure the characteristics of the material isolating one of the two processes. Furthermore, in most of the index modulated holographic recording materials, there is also some residual surface relieve modulation. In some cases, this thickness variation may represent a large percentage of the phase modulation introduced by the material onto the incident beam. Usually shrinkages around $8 \%$ are reported in the literature some polymer formulations ${ }^{16-17}$. Shrinkage of $0.5 \%$ is defined as the upper limit for commercial availability of a recording medium ${ }^{18-19}$, on the other hand it is interesting to obtain relief profiles for certain applications ${ }^{20}$. Usually the contribution of the index and thickness variations are not easy to decouple. Recently, we have proposed the application of interferometric techniques, both in transmission ${ }^{21}$ and in reflection ${ }^{22}$, to characterize in realtime the modulation performance of the photopolymers. Interferometric techniques are a well established tool in many metrological applications. However, they have rarely been applied in the realm of holographic recording materials, and more specifically in the development of photopolymeric media. These interferometric techniques allow both to obtain a direct measurement for the index modulation and for the thickness variation.

In the last ten years, some studies have been carried out dealing with the recording of very low spatial frequencies (less than 10 lines/mm) in PVA/AA based layers. In Ref. [23] a contact-copying process was used to transfer low spatial frequency diffractive optical elements (DOEs) from binary amplitude masks ${ }^{24}$ onto the photopolymer, with good results. The same technique was used to analyze the suitability of a wide range of holographic recording materials at low spatial frequencies so as to register DOEs and optical correlation filter ${ }^{25}$. Recently, we proposed a hybrid optical-digital setup incorporating a liquid crystal display (LCD) to generate phase DOEs onto photopolymers ${ }^{26}$. The application of photopolymers to DOEs generation requires the characterization of the modulation properties of the material. The interferometric techniques proposed in Ref. [21,22] are well adapted for this characterization, since they measure the phase-shift modulation in the range of the very low frequencies usually registered in DOEs.

In this work we show the application of interferometric measurements to characterize the modulation properties of photopolymers. A double beam interferometer is constructed, both in transmission and in reflection, in combination with the setup to expose the recording material. Some benefits are provided by this approach: direct calculation of the properties of the material is possible, index and surface modulation can be decoupled, and additional information can be obtained since the results are not influenced by diffusion processes. With this scheme we mainly characterize the properties at very low spatial frequencies, which can be useful to analyze the applicability of holographic recording materials in another range of applications, such as recording of diffractive optical elements (DOEs). Comparison with the conventional holographic characterization shows significant differences. In Section 2 we describe the recording setup where an additional arm is added for the interferometric measurements. In Section 3 we show the results for the phaseshift and for the shrinkage as a function of the exposure obtained for various compositions and thicknesses of the layers of PVA/AA photopolymer. The main conclusions of the work are given in Section 4.

\section{COMBINED RECORDING SETUP WITH A REAL TIME INTERFEROMETER}

In this Section we focus on the description of the combined setup to obtain real time interferometric measurements while the holographic material is being exposed. First we describe the combined setup with the interferometer in transmission. Then we describe the specific details for the interferometer in reflection.

In Figure 1 we show the experimental setup in transmission geometry to measure the phase-shift as a function of the exposure energy. The setup has two arms with an angular separation of $30^{\circ}$, one to expose the recording material, whereas the second arm is the interferometer used to measure, in real-time, the phase-shift. The recording material is perpendicularly oriented with respect to the interferometer axis in order to ease the analysis of the interferometric results: at an oblique incidence we should take into account both the Fresnel coefficients at the interface and the increase of distance in the propagation across the layer.

In the first arm, the exposure beam provided by a solid-state Nd-YVO4 Verdi laser with a wavelength of $532 \mathrm{~nm}$ (at this wavelength the dye presents the maximum absorption) is expanded and collimated using a spatial filter and a lens, obtaining a beam with $1.5 \mathrm{~cm}$ of radius. A wave plate and a neutral filter (attenuator) are added before the spatial filter to 
control the orientation and the intensity of the linearly polarized beam produced by the laser Nd-YVO4. A polarizer (P), with its transmission axis oriented along the vertical of the lab, is introduced to produce a beam with TE polarization incident onto the recording material. This incident beam forms an angle of $30^{\circ}$ with respect to the photopolymer layer. We adjust the laser power so that the exposure intensity that impinges on the layer is $0.4 \mathrm{~mW} / \mathrm{cm}^{2}$ (this is the value corrected from the Fresnel coefficient at the air-photopolymer interface at an incidence of $30^{\circ}$ for TE polarization). A half-opened diaphragm is used to leave an unexposed area in the photopolymer layer.

In the interferometric arm, to generate the interferences pattern we use a He-Ne laser, since the photopolymer does not present any absorption at $633 \mathrm{~nm}$. We have implemented a Young's fringes based two beams interferometer. This interferometer has been successfully applied in the phase-shift characterization of liquid crystal displays (LCDs) ${ }^{27}$. It shows a good precision, and due to its quasi-common path architecture is a robust setup, less sensible to changing environmental conditions and simpler to construct than Mach-Zehnder type interferometers. We use a grating with a spatial frequency of 4 lines $/ \mathrm{mm}$ to generate a series of diffracted orders from the unexpanded He-Ne beam; we block all the orders except -1 and +1 . One of the two orders impinges on the exposed zone (illuminated by the Nd-YVO4 laser) and the other one impinges on the non-exposed zone. The distance between the two orders is about $1 \mathrm{~cm}$, so as to eliminate the influence of the monomer diffusion in the polymerization process. Once the two orders have propagated throughout the photopolymer, a lens is used to make them interfere. A microscope objective is used to amplify the interference pattern onto a CCD camera. This pattern is captured in real-time as a function of exposure at specific time intervals. The measurements and the results obtained using this setup are presented in Section 3.1.

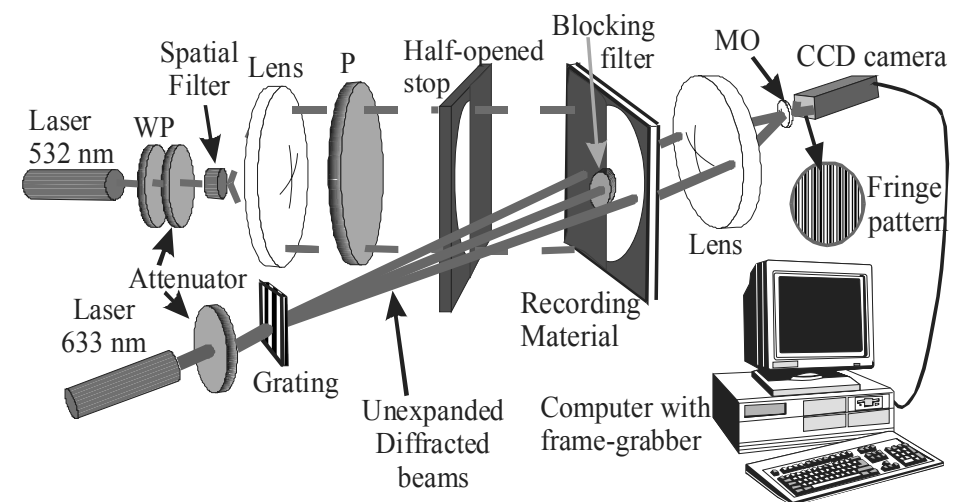

Figure 1. Experimental setup. The recording material is exposed with the green laser beam $(\lambda=532 \mathrm{~nm})$ and the phase-shift is measured with the red beam $(\lambda=633 \mathrm{~nm})$. P is polarizer, WP is wave plate, MO is microscope objective.

The scheme for the setup to measure the phase-shift in reflection is basically the same as in Figure 1. Instead of measuring the interference between the two transmitted beams, the measurements are done for the reflected beams. The interferometric arm is, thus, located on the same side of the incident He-Ne probe beam at an angle, so as to separate the incident and reflected He-Ne beams. With these measurements the thickness variation, thus the shrinkage, can be measure as a function of the exposure energy. Results obtained are shown in Section 3.2.

In Figure 2 we show as an example the interference fringes captured at 4 different exposure times. We see how the fringes shift while the exposure time increases. The patterns show a very good visibility (both in images captured in the transmission and in the reflection interferometers). This implies that neither the amplitude transmission nor the reflection coefficient vary with the exposure. Actually the material can be considered transparent to the $633 \mathrm{~nm}$ wavelength. Therefore, with this setup it is possible to plot the fringes moving as a function of the exposure time during the polymerization process, and without the influence of monomer diffusion. In this sense, we have verified that once we stop the exposition, the shift in the interference fringes freezes. This indicates that the measurements taken in this setup are not affected by diffusion processes. 


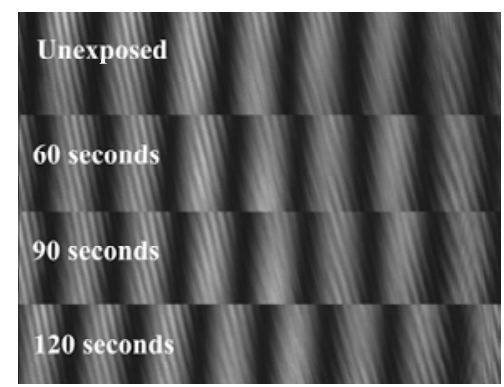

Figure 2. Fringe pattern for different exposure times (before exposing the material, after $60 \mathrm{~s}, 90 \mathrm{~s}$ and $120 \mathrm{~s}$ respectively). In particular, these images correspond to material 2 (see Table 1).

Once the interference pattern has been stored, we measure the shift with respect to the initial pattern obtained for the unexposed layer. To increase the accuracy in this calculation, we cross-correlate the different interference patterns with respect to the unexposed ones. The cross-correlation produces a clear peak. The location of this peak for each exposure with respect to the center of the image is equal to the shift in the fringe pattern. A full fringe shift is equal to a $2 \pi$ radians variation in the phase-shift. In this experiment, the phase-shift is directly related to the index modulation and/or the thickness modulation of the material, depending if we analyze the measurements obtained with the transmission or the reflection interferometer. This simple and direct relation is not possible in the case of the holographic characterization where multiparametric diffusion models are used.

\section{RESULTS FOR THE MODULATION CAPABILITIES OF THE PHOTOPOLYMER}

\subsection{Photopolymer layers}

The PVA/AA formulations contain a dye, a cosensitizer wich is triethanolamine (TEA), one or two monomers (AA and or not BMA) and a polymer (PVA). Let us introduce some basic details related to the design of the composition of PVA/AA photopolymer layers. Since the molecular weight of the PVA plays an important role in the monomer diffusion during the polymerization process ${ }^{28}$, different types of PVA have been used in the literature. Moreover, different dyes and different triethanolamine (TEA) concentrations are normally used to obtain different material properties (TEA is a liquid at ambient temperature and plays an important role in the monomer and polymer diffusion during the polymerization process too ${ }^{12}$ ).

Table 1. Water solutions used to obtain phopopolymerizable "dry" layers.

\begin{tabular}{|l|llll|}
\hline Component & \multicolumn{2}{|l|}{ Type 1 } & \multicolumn{2}{|l|}{ Type 2 } \\
\hline $\begin{array}{l}\text { PVA } \\
\mathbf{M}_{\mathbf{w}}=\mathbf{1 3 0 0 0 0}\end{array}$ & $8.5 \%$ & $\mathrm{w} / \mathrm{v}$ & $8.5 \%$ & $\mathrm{w} / \mathrm{v}$ \\
\hline TEA & 0.4 & $\mathrm{M}$ & 0.4 & $\mathrm{M}$ \\
\hline YE & $2.5 \times 10^{-4}$ & $\mathrm{M}$ & $2.5 \times 10^{-4}$ & $\mathrm{M}$ \\
\hline AA & 0.45 & $\mathrm{M}$ & 0.40 & $\mathrm{M}$ \\
\hline BMA & \multicolumn{2}{|c|}{ Non } & 0.05 & $\mathrm{M}$ \\
\hline
\end{tabular}

In this work, we consider PVA with a molecular weight of 130000, and the TEA concentration is varied between $0.4 \mathrm{M}$ and $0.15 \mathrm{M}$ (see Table 1). The photopolymerizable solution is prepared mixing yellowish eosin (YE, the dye), together with a mixture of acrylamide (the monomer) and triethanolamine (the co-initiator), and adding the PVA (the binder). In this study the solutions are prepared using a conventional magnetic stirrer, under red light and in standard laboratory conditions (temperature, pressure, relative humidity). The solutions are deposited, by gravity, over glasses (size $20 \times 40$ $\mathrm{cm}^{2}$ ). Afterwards the solutions are left in the dark to allow the water evaporation. When a high percentage of the water content has already evaporated ${ }^{11}$, the material is cut into squares $\left(6.5 \times 6.5 \mathrm{~cm}^{2}\right)$ using a glass cutter. The water solutions used in this paper are presented in Table 1. Compositions type 1 and 2 are used to obtain layers with thickness between 
60 and $120 \mu \mathrm{m}$; in the case of composition type 2 the BMA is included in the solution ${ }^{29}$. Analysis of thick layers is undertaken in Ref. [21]. In reflection we have studied the influence of BMA (crosslinker) in the shrinkage of the layers.

\subsection{Results in transmission}

We present the experimental data obtained for different material compositions and different thicknesses. The size of the error bars included in the figures indicates the repeatability or our experiments. This repeatability decreases when there are variations of the humidity and temperature in our laboratory during the drying process.

Let us begin the analysis with the basic material composition (type 1). In Figure 3 we show the phase-shift between the bright and dark zones as a function of the exposure time for material type 1; these layers have a thickness of $70 \mu \mathrm{m}$. We see that after an exposure of $400 \mathrm{~s}\left(160 \mathrm{~mJ} / \mathrm{cm}^{2}\right)$, the material is in the saturation region and no more changes can be observed: it is possible to assume that the polymerization process is finished. The saturation energy value is in the range obtained when characterizing the material at high spatial frequencies (about $120 \mathrm{~mJ} / \mathrm{cm}^{2}$ ). The maximum phase-shift value is around $192^{\circ}$; this value is sufficient in order to store binary-phase DOEs but it is too small to record non-binary DOEs, as diffractive lenses, blazed gratings or kinoforms, where a $360^{\circ}$ phase-shift depth is generally required ${ }^{30}$.

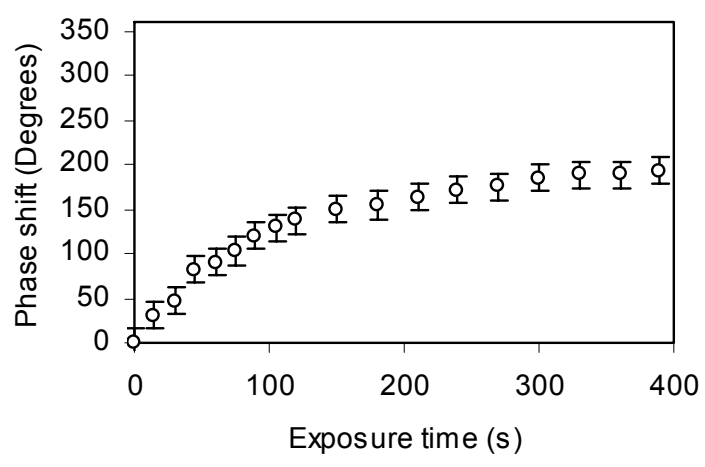

Figure 3. Phase-shift as a function of the exposure time for layers type 1 and $70 \mu \mathrm{m}$ thick.

In order to achieve higher values of phase-shift, there are two possibilities: to increase the thickness or to increase the refractive index modulation. In order to increase the refractive index, we can use crosslinker monomers as BMA. In Figure 4(a) we present the results provided for material type 2 (for the same thickness of material type $1,70 \mu \mathrm{m}$ ). In Figure 4(b) we present the results provided for material type 2 with a thickness of $115 \mu \mathrm{m}$. It is possible to observe that if we use BMA in the chemical material composition, we obtain higher values of the phase-shift. Particularly, in Figure 4.b we achieve phase shifts significantly larger than $360^{\circ}$ : that is, we can use this material to store non-binary DOEs as lenses. The only important disavantage is the non-linearity between exposure time and phase-shift. Moreover, it is important to note the difference between the saturation exposure times for material type 1 (without BMA), shown in Figure 1, and type 2 (with BMA). Material type 1 presents saturation exposure times around $300 \mathrm{~s}$ whereas in the case of material type 2 these times are around 200 seconds. This effect clearly shows the increase in the polymerization rate when BMA is present ${ }^{29}$. 


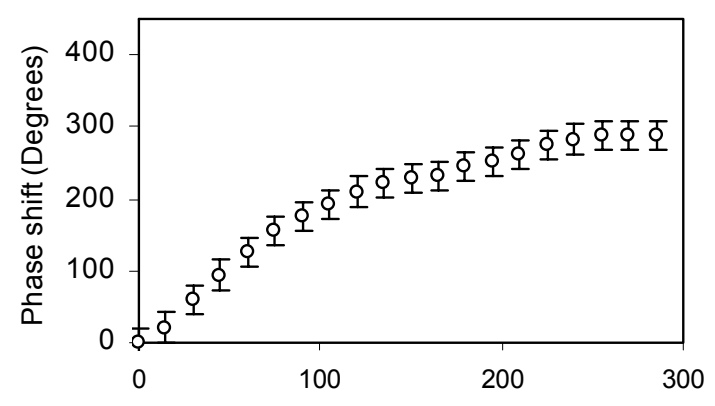

(a)

Exposure time (s)

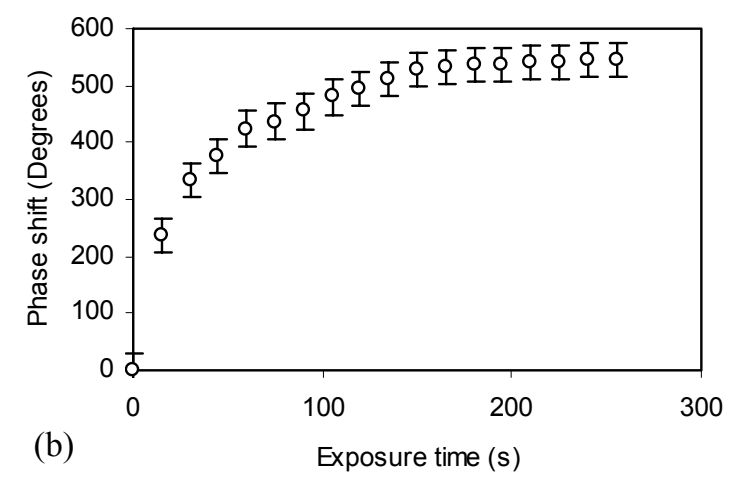

Figure 4. Phase shift as a function of the exposure time for layers type 2: (a) for $70 \mu \mathrm{m}$ thick and (b) for $115 \mu \mathrm{m}$ thick.

Eventually, once we have characterized the different layers, it is important to remark that the huge phase-shift between the bright and dark zones cannot only be explained by a refractive index modulation, that is, a certain degree of thickness variation between the bright and dark regions should clearly contribute to the phase-shift. If we assume that there is no thickness variation, the saturation refractive index modulation should be around $5 \times 10^{-3}$ for material type 1 , and $8 \times 10^{-3}$ for material type 2. Taking into account these results, the values are $30 \%$ higher than the typical values of refractive index modulation obtained in the holographic range where the spatial frequencies are around 1000 lines $/ \mathrm{mm}$. Furthermore, in the case of the holographic high spatial frequencies, there is an important contribution of free monomer coming from the non-exposed zones (monomer diffusion plays an important role for high spatial frequencies), which contributes to increase the refractive index modulation. These results suggest that PVA/AA based materials present a swelling or a shrinkage during the polymerization process in the experiments carried out. In this sense it is well known that volume shrinkage acts during the homogeneous polymerization of polymers ${ }^{16,17}$. In the next Section we focus on the study of the thickness variations, using the phase-shift measurements obtained with the reflection type interferometer.

\subsection{Results in reflection}

We analyze the thickness variation for two different compositions, with and without crosslinker, at zero frequency. Once obtained the phase shift between exposed and non exposed zones as a function of the exposure with the reflection interferometer, the shrinkage of the layer during exposure can be directly calculated using the following expression,

$$
\Delta d=\frac{\Delta \Phi \lambda \cos \alpha}{4 \pi}
$$

where $\Delta d$ is the thickness variation, $\Delta \Phi$ is the phase-shift between exposed and non-exposed zones (expressed in radians), $\lambda$ and $\alpha$ are the wavelength and the incident angle of the reading beam respectively.

\subsubsection{Layers without crosslinker}

The layers without crosslinker are characterized by shorter polymer chains, less energetic sensitivity, higher values of inhibition period ${ }^{31-33}$ and low values of diffraction efficiency when this type of layers are used in holography. In Figure 5(a) we show the phase shift between exposed and non exposed zones as a function of the exposure measured with the reflection type interferometer. Using Eq. (1) we calculate the shrinkage of the layer during exposure. We show the results in Figure 5(b). The shrinkage suffered by the sample during exposition is around $2 \mu \mathrm{m}$ after 300 seconds. Due to this effect appears a variation of the Bragg angle in holographic application (i.e. when slanted grating are stored). Note that this shrinkage value exceeds the $0.5 \%$ defined as the upper limit for commercial availability of a recording medium ${ }^{18-19}$. 

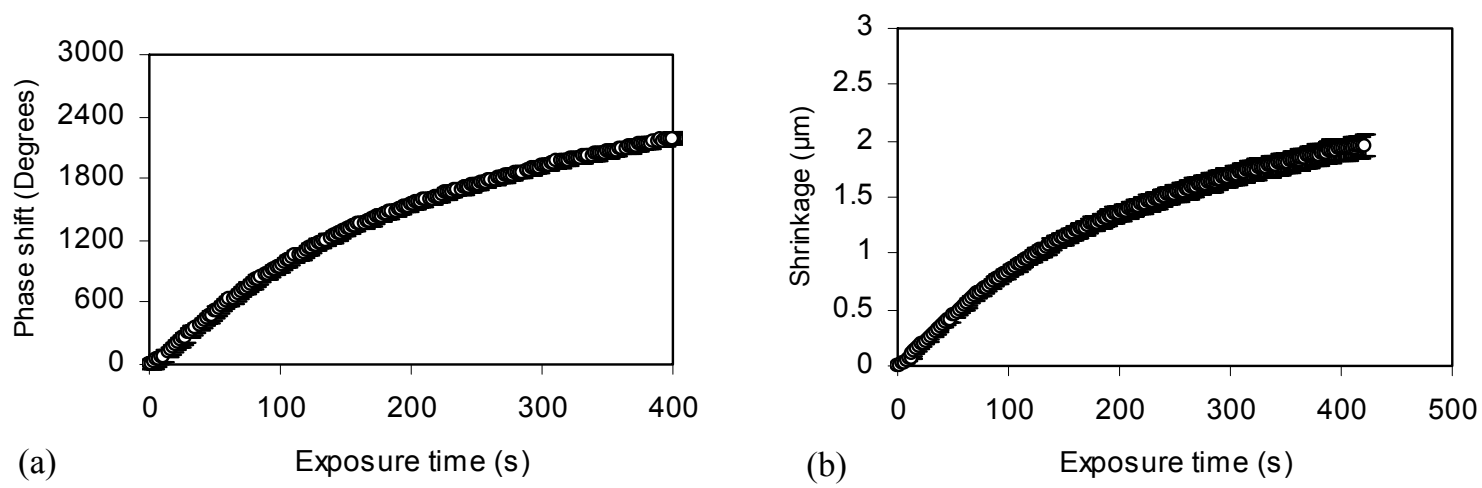

Figure 5. Layers without crosslinker. (a) Phase-shift as a function of the exposure time measured with the reflection interferometer. (b) Shrinkage as a function of the exposure time.

\subsubsection{Layers without crosslinker}

Next we study the influence of the crosslinker in the material behaviour. In our experiments we use BMA as crosslinker. It is well known the capability of a crosslinker to increase the refractive index modulation of the polymer, the energetic sensitivity and the polymer length chains. On the other hand it is interesting to analyze the influence of the crosslinker in the layer shrinkage. We present the results for the phase shift as a function of exposure time in Figure 6(a). It is interesting to note the high values obtained in this case, due to the higher compaction produced by the BMA. Now the shrinkage is $33 \%$ higher than the case without crosslinker $(3 \mu \mathrm{m})$ (Figure 6(b)).
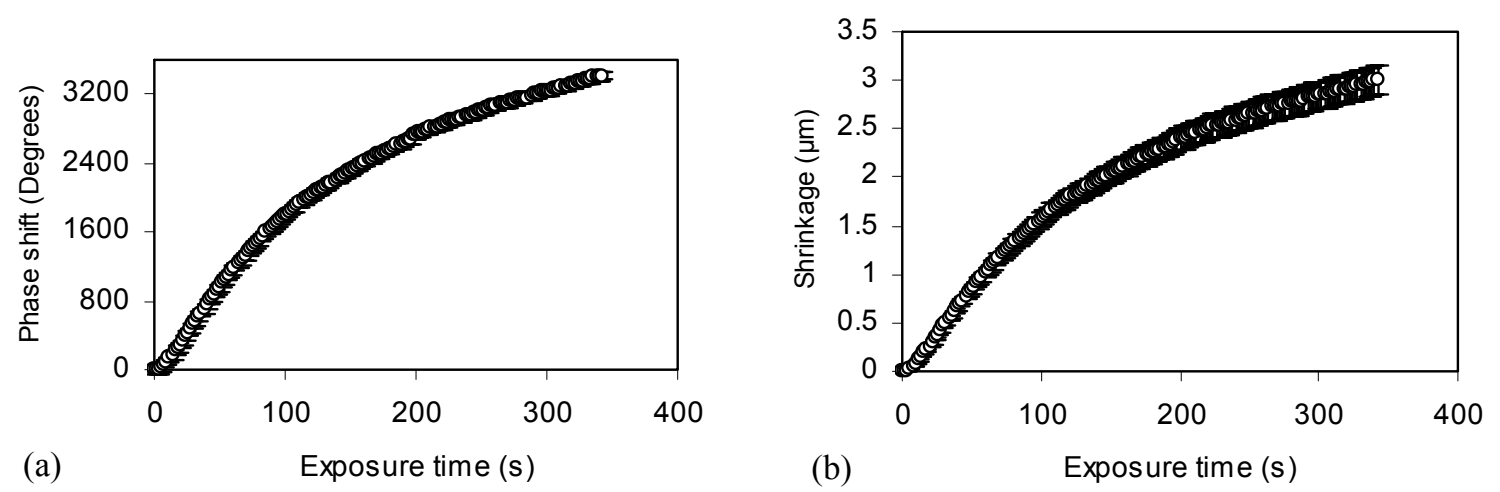

Figure 6. Layers with crosslinker. (a) Phase-shift as a function of the exposure time measured with the reflection interferometer. (b) Shrinkage as a function of the exposure time.

\section{CONCLUSIONS}

In this work we have characterized the optical modulation properties of PVA/AA based layers with different compositions and thicknesses. A novel technique based on a Youngs's based double beam interferometer is proposed, which allows obtaining in real-time direct values for the phase-shift modulation of the recording material as a function of exposure. The characterization technique proposed does not depend on monomer diffusion processes, since a uniform exposure (zero frequency) is recorded onto the material. From the point of view of the phase-shift range depth, we have demonstrated the capability of some layer compositions to store phase elements under red illumination. We have achieved large phase-shifts between exposed and non-exposed zones exceeding $360^{\circ}$. Using the interferometer in reflection we have calculated the shrinkage for two compositions with and without BMA as crosslinker at zero spatial 
frequency limit. We have calculated values of shrinkage about $2 \%$ (without crosslinker) and $3 \%$ (with crosslinker) in the layers analyzed, these values show a potential for the application to relief structures on the photopolymer, on the other hand the shrinkage is too high for holographic applications.

\section{ACKNOWLEDGMENTS}

We acknowledge financial support from the Spanish Ministerio de Educación y Ciencia (grants FIS2005-05881-C02-01 and FIS2005-05881-C02-02), and from Generalitat Valenciana (grant GV06-007).

\section{REFERENCES}

1. A. Pu, D. Psaltis, "High-density recording in photopolymer-based holographic three-dimensional disks", Appl. Opt. 35, 2389-2398, (1996).

2. Y. Tomita, K. Furushima, K. Ochi, K. Ishizu, A. Tanaka, M. Ozawa, M. Hidaka, and K. Chikama, "Organic nanoparticle (hyperbranched polymer)-dispersed photopolymers for volume holographic storage," Appl. Phys. Lett. 88, 071103 (2006).

3. A. Márquez, C. Neipp, S. Gallego, M. Ortuño, A. Beléndez and I. Pascual, "Edge enhanced imaging using PVA/acrylamide photopolymer gratings", Opt. Lett. 28(17), 1510-1512 (2003).

4. S. M. Schultz, E. N. Glytsis, and T. K. Gaylord, "Design of high-efficiency volume gratings couplers for line focusing," Appl. Opt. 37, 2278-2287 (1998).

5. D. A. Waldman, C. J. Butler, and D. H Raguin, "CROP holographic storage media for optical data storage at grater

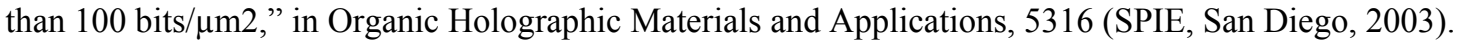

6. W.L. Wilson, K.R. Curtis, K. Anderson, M. C. Tackitt, A. J. Hill, M. Pane, C. Stanhope, T. Earhart, W. Loechel, C. Bergman, K. Wolfgang, C. Shuman, G. Hertrich, K. Parris, K. Malang, B. Riley and M. Ayer, "Realization of high performance holographic data storage: The inPhase Technologies demonstration platform," Proc. SPIE 5216, 178191, (2003).

7. H. J. Coufal, D. Psaltis, and G. T. Sincerbox, eds., Holographic Data Storage, Springer Series in Optical Sciences, (Springer-Verlag, Berlin, 2000).

8. C. Neipp, A. Beléndez, S. Gallego, M. Ortuño, I. Pascual and J. T. Sheridan, "Angular responses of the first and second diffracted orders in transmission diffraction grating recorded on photopolymer material," Optics Express 11, 1835-1843 (2003).

9. J. V. Kelly, F. T. O' Neill, C. Neipp, S. Gallego and M. Ortuño and J. T. Sheridan, "Holographic photopolymer materials: non-local polymerisation driven diffusion under non-ideal kinetic conditions" J. Opt. Soc. of Am. B 22, 22, 407-406 (2005).

10. S. Martin, C. A. Feely and V. Toal, "Holographic recording characteristics of an acrylamide-based photopolymer", Applied Optics 36, 5757-5768 (1994).

11. M. Ortuño, S. Gallego , C. García , C. Neipp , A. Beléndez and I. Pascual "Optimization of a $1 \mathrm{~mm}$ thick PVA/acrylamide recording material to obtain holographic memories: method of preparation and holographic properties" Applied Physics B, 76, 851-857 (2003).

12. S. Gallego, M. Ortuño, C. Neipp, A. Márquez, A. Beléndez, I. Pascual, "Characterization of polyvinyl alcoholacrylamide holographic memories with a first-harmonic diffusion model" Appl. Opt. 44, 6205-6210 (2005)

13. S. Gallego, C. Neipp, M. Ortuño, A. Márquez, A. Beléndez, I. Pascual "Diffusion based model to predict the conservation of holographic gratings recorded in PVA/Acrylamide photopolymer," Appl. Opt. 42, 5839-5845 (2003).

14. A. Márquez, C. Neipp, S. Gallego, M. Ortuño, A. Beléndez and I. Pascual, "Holographic optical elements for Bragg image processing", Proc. SPIE 5827, 152-162 (2005).

15. G. Zhao and P. Mouroulis, "Diffusion model of hologram formation in dry photopolymers materials," J. Mod. Opt. 41, 1929-1939 (1994).

16. J.E. Dietz, N.A. Peppas, "Reaction kinetics and chemical changes during polymerization of multifunctional (meth)acrylates for the production of highly crosslinked polymers used in information storage systems", Polymer, 38 (15), 3767-81, 1997 
17. T. Endo, F. Sanda, "Ring-opening polymerization, anionic (with expansion in volume)", Polymeric Materials Encyclopedia, 10, CRC Press, Inc.,7550-3, 1996

18. G. Ramos, A. Álvarez-Herrero, T. Belenguer, F. del Monte, and D. Levy, "Shrinkage Control in a Photopolymerizable Hybrid Solgel Material for Holographic Recording," Appl. Opt. 43, 4018-4024 (2004).

19. L. Dhar, M. G. Schones, T. L. Wysocki, H. Bair, M. Schilling,and C. Boyd, "Temperature-induced changes in photopolymervolume holograms," Appl. Phys. Lett. 73, 1337-1339 1998.

20. I. Naydenova, E. Mihaylova, S. Martin, and V. Toal, "Holographic patterning of acrylamide-based photopolymer surface," Opt. Express 13, 4878-4889 (2005)

21. S. Gallego, A. Márquez, D. Méndez, M. Ortuño, C. Neipp, M. L. Alvarez, A. Beléndez, E. Fernández and I. Pascual, "Real-time interferometric characterization of a PVA based photopolymer at the zero spatial frequency limit” Appl. Opt. 46, 7506-7512 (2007).

22. S. Gallego, A. Márquez, D. Méndez, M. Ortuño, C. Neipp, M. L. Alvarez, A. Beléndez, E. Fernández and I. Pascual, "Analysis of PVA/AA based photopolymers at the zero spatial frequency limit using interferometric methods" (manuscript in preparation for Applied Optics).

23. I. Pascual, A. Márquez, A. Beléndez, A. Fimia, J. Campos and M. J. Yzuel, "Copying low spatial frequency diffraction gratings in photopolymer as phase holograms", J. Mod. Opt. 47, 1089-1097 (2000).

24. A. Márquez, J. Campos, M. J. Yzuel, I. Pascual, A. Fimia and A. Beléndez, "Production of computer-generated phase holograms using graphic devices: application to correlation filters", Opt. Eng. 39, 1612-1619 (2000).

25. A. Márquez, C. Neipp, A. Beléndez, J. Campos, I. Pascual, M. J. Yzuel and A. Fimia, "Low spatial frequency characterization of holographic recording materials applied to correlation”, J. Opt. A: Pure Appl. Opt. 5, S175-S182 (2003).

26. A. Márquez, S. Gallego, D. Méndez, M. L. Álvarez, E. Fernández, M. Ortuño, C. Neipp, A. Beléndez, I. Pascual, "Application of a liquid crystal display to generate diffractive optical elements onto a photopolymer", Proc. EOS Topical Meeting on Diffractive Optics, 194-195 (2007).

27. A. Bergeron, J. Gauvin, F. Gagnon, D. Gingras, H. H. Arsenault and M. Doucet, "Phase calibration and applications of a liquid-crystal spatial light modulator", Appl. Opt. 34, 5133-5139 (1995).

28. V. Weiss, E. Millul y A. A. Freisem, "Improvements in holographic photopolymers at Weizmann Institute of Science", SPIE International Technical Group Newsletter: Optics in Information Systems 15, No 1, 3 (2004).

29. Cristian Neipp, Sergi Gallego, Manuel Ortuño, Andrés Márquez, Augusto Beléndez, Inmaculada Pascual "Characterization of a PVA/acrylamide photopolymer. Influence of a cross-linking monomer in the final characteristics of the hologram" Opt. Commun., 224, 27-34 (2003).

30. D. C. O'Shea, T. J. Suleski, A. D. Kathman and D. W. Prather, Diffractive Optics: design, fabrication and test, SPIE (2004).

31. P. J. Flory, Principles of Polymer Chemistry, Cornell U. Press, Ithaca, N.Y., 1953, pp. 161-177.

32. Ortuño, S. Gallego, C. García, C. Neipp, I. Pascual "Holographic characteristics of a $1 \mathrm{~mm}$ thick photopolymer to be used in holographic memories" Appl. Opt. 42, 7008-7012 (2003).

33. M. R. Gleeson, J. V. Kelly, C. E. Close, F. T. O’Neill, J. T. Sheridan, "The Impact of Inhibition Processes during Grating Formation in Photopolymer Materials", in Photonic Engineering, R. F. O'Dowd, ed., Proc. SPIE 5827, (2005). 